\title{
Longitudinal fibre splitting in muscular dystrophy: a serial cinematographic study
}

\author{
EDWARD R. ISAACS, ${ }^{1}$ WALTER G. BRADLEY ${ }^{2}$, AND GERALD HENDERSON \\ From the Muscular Dystrophy Group Research Laboratories, \\ Newcastle General Hospital, Newcastle upon Tyne
}

SUMMARY A technique of block surface-staining and serial cinematography was modified to review serial sections of normal and dystrophic muscle from the Bar Harbor $129 \mathrm{Re}$ strain of mice as a preliminary study of fibre splitting in dystrophic muscle. Using this technique, muscle fibres were reconstructed for up to $1.5 \mathrm{~mm}$ of their length without difficulty. Split fibres were identified only when the actual separation of fibres was observed. Splitting was seen to be a significant cause of the variations in fibre diameter and was at times responsible for the formation of groups of small atrophic fibres which resembled those seen in denervation atrophy. Complex multiple splitting and recombination of daughter and parent fibres was also observed and reconstructed to scale. These results may have considerable significance for the interpretation of physiological data on both human and murine dystrophic muscle.

The longitudinal splitting of muscle fibres is one of the degenerative changes which may be identified in transverse sections of muscle tissue. Daughter fibres share a common endomysial sheath but possess their own sarcolemmal membrane (Adams, Denny-Brown, and Pearson, 1962). Central migration of sarcolemmal nuclei appears to precede the longitudinal separation of the sarcoplasm (Banker, 1960). Usually, from two to five daughter fibres can be delineated in a split fibre.

One of the underlying causes for splitting seems to be related to increased functional stress (Edgerton, 1970; Hall-Craggs, 1970). It is described in chronic neurogenic disorders (Pearce and Harriman, 1966; Drachman Murphy, Nigam, and Hills, 1967) though not in the more acute forms, perhaps as a result of a 'functional overload' in some of the remaining, innervated, hypertrophied fibres (Cazzato, 1970). In Duchenne type muscular dystrophy the incidence of splitting is lowest in the early and preclinical stages, increases to a maximum in the

\footnotetext{
1 Present address: Division of Neurology, Medical College of Virginia, Health Sciences Division, Virginia Commonwealth University, 1200 East Broad Street, Richmond, Virginia 23219, U.S.A.

2 Reprint requests to W.G.B.
}

moderately affected stage, and is significantly lower in the more severely handicapped patient confined to a wheelchair (Bell and Conen, 1968). In murine muscular dystrophy, animals which are 'too sickly' for sale to research laboratories, and which therefore have a more severe disease show no splitting (West and Murphy, 1960).

The full extent and complexities of fibre splitting have never been completely described. As a preliminary approach to this problem, a technique of serial cinematography (Hegre and Brashear, 1947; Hegre, 1951; Read, Hegre, and Russi, 1953; Hegre, 1967) was modified and adapted as a unique method to view and study relatively large numbers of serial sections of both normal and dystrophic muscle. In this preliminary study, muscle from mice with muscular dystrophy was studied.

\section{METHODS}

Portions of gracilis muscle were obtained from 8-12 weeks old normal and dystrophic mice of the Bar Harbor $129 \operatorname{Re}$ strain. The specimens were placed in isotonic saline for 6-8 hours, allowing the muscle fibres to relax and separate. The tissue was then fixed in Susa and double embedded using a modified Peterfi technique. The muscle was embedded in 


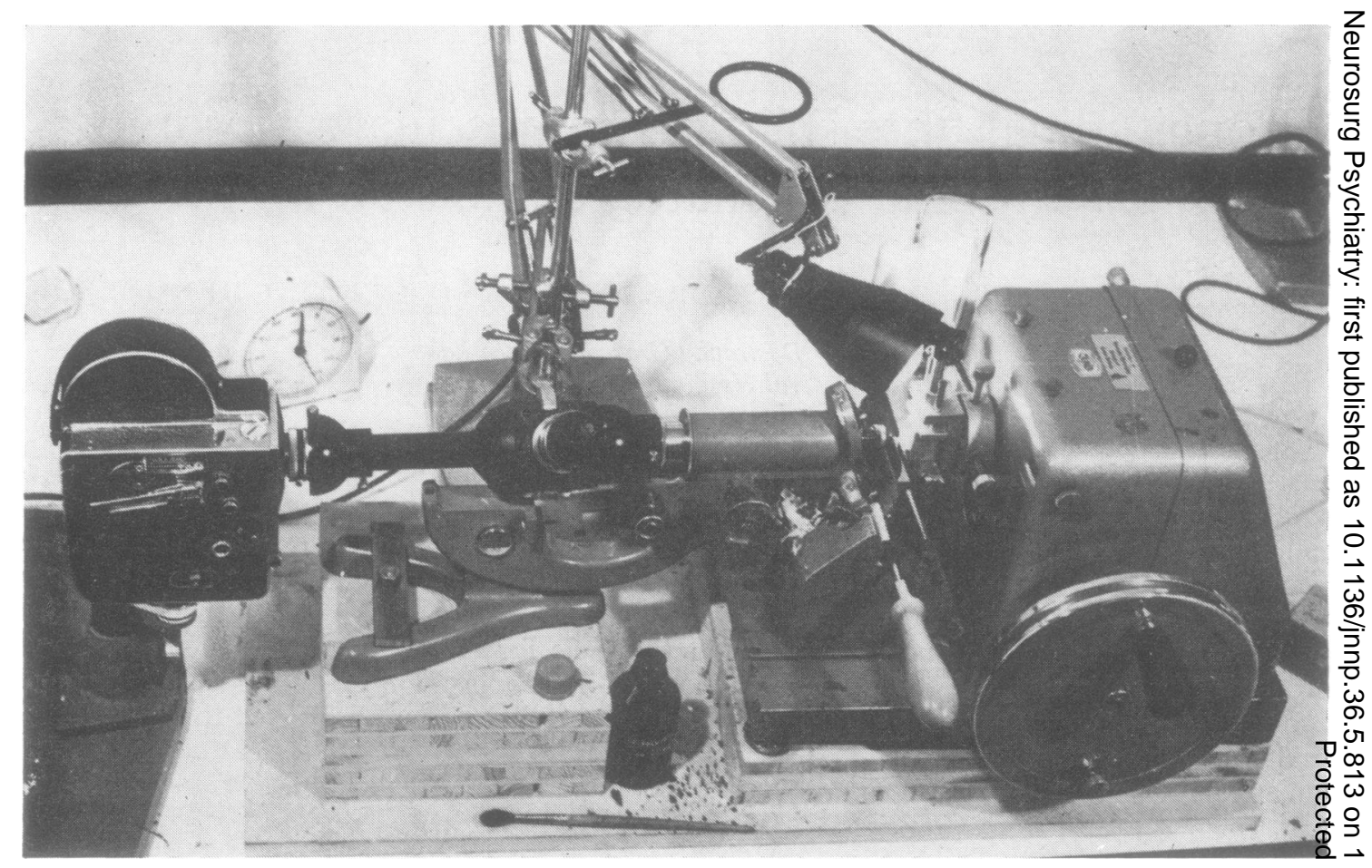

FIG. 1. Photograph of the apparatus (see text).

Paraplast and orientated to give transverse sections. An apparatus was assembled by mounting and aligning a camera, microscope and a microtome. The microtome used was Jung 1130 in which the block returns to, and is locked in, the same position after each cutting stroke. This facility is not found in a sliding microtome where the position of the block shifts, nor in the Beck rotary microtome where the knife moves toward the block and the position of the block surface is changed with each stroke. A Beck/ London monocular microscope was modified to observe the block face, with the body in the inverted position. The $\times 10$ objective was used to observe the cut surface of the block. The mirror, sub-stage condenser, and stage were removed to allow a reflex viewing system to be introduced. The film was exposed using a Paillard/Bolex $16 \mathrm{~mm}$ cine camera with a Yver 1.8 lens (Fig. 1). The final magnification of this optical system was $\times 12$. The surface of the block was illuminated by incident light using a 250 watt bulb; overheating was avoided by using a cooling fan.

Once trimmed, the surface of the block was coated with $1 \%$ Toluidine Blue in $1 \%$ borax for $10-15$ seconds, then blotted dry. An area of the tissue was selected by minor angle adjustments on the micro tome, and the microscope focused. After the camera. was set to the maximal aperture, and the focal length to infinity, the film was exposed for one second. A $10 \mu$ section was cut, the surface re-stained and blotted, and another exposure made. This was repeated, with minor adjustments in focusing and $\stackrel{\complement}{\complement}$ angulation when necessary, until all of the tissue in $\overrightarrow{\overrightarrow{0}}$ the block was cut.

After developing the film, it could be viewed with a slow-motion or single frame projector. For furthero detailed analysis and reconstruction of the muscle fibres, segments of the film were reproduced frame by frame using a $3 \mathrm{M}$ ' 400 ' Reader-Printer photo- -0 copier on 3M microfilm print paper type 769 . Muscle fibres were then identified by numbers on each serial print. Since each print represented a 3 thickness of $10 \mu$, selected muscle fibres could be reconstructed to scale (Figs 2 and 3).

\section{RESULTS}

Muscle fibre splitting was not observed in muscle from normal control mice.

In dystrophic mouse muscle, a number of fibres 

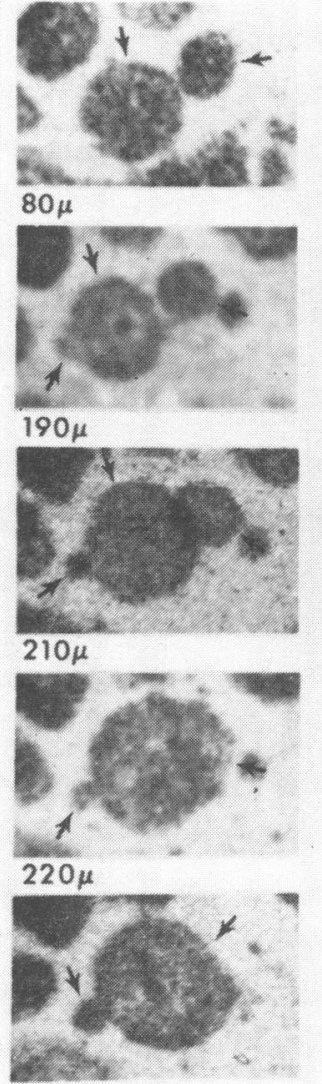

$240 \mu$

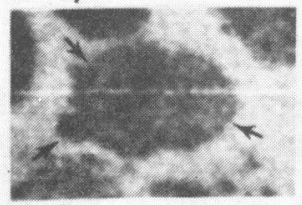

$340 \mu$

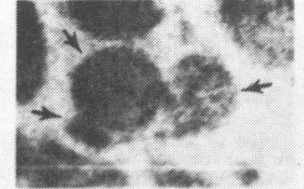

$370 \mu$

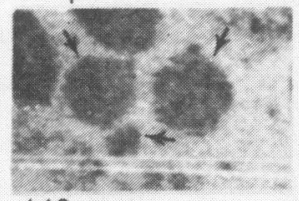

\section{$440 \mu$}
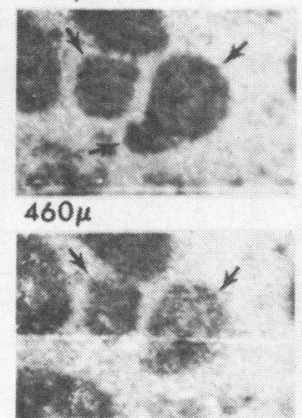

$470 \mu$

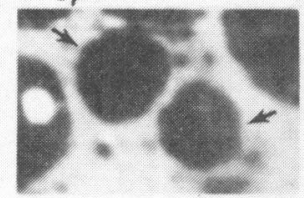

$590 \mu$

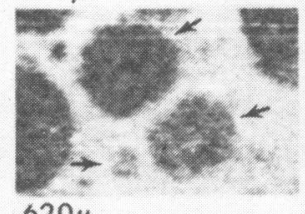

$620 \mu$

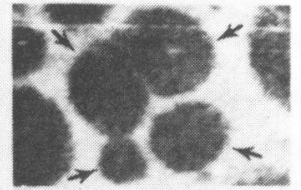

$740 \mu$

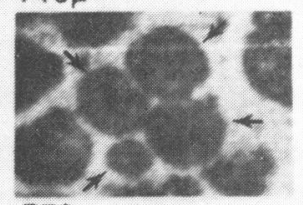

$770 \mu$

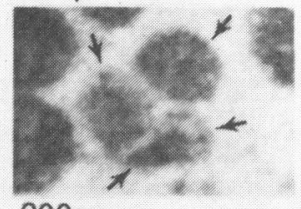

$900 \mu$

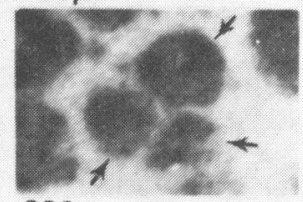

$920 \mu$

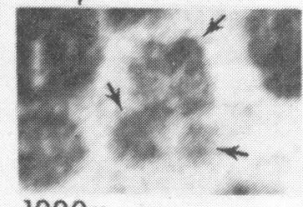

$1000 \mu$

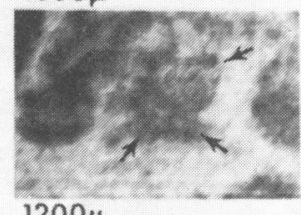

$1200 \mu$
FIG. 2. Selected sections obtained from prints of individual frames showing the actual appearance of one of the fibres at various levels representing key points in its reconstruction. were noted to split longitudinally. Daughter or split fibres were identified only when an actual separation from the parent fibre was seen, and when the daughter fibre could be clearly and consistently identified. Usually, these fibres were at least $10 \mu$ in diameter and separated from the parent fibre for a distance of at least $50 \mu$. Using these criteria the following observations were made:

1. In an area of about 430,000 square microns, containing approximately 100 muscle fibres, 64 fibres were consistently identified in serial sec- tions. Of these, 12 fibres, ranging from $14 \mu$ to $60 \mu$ in diameter, were seen to split.

2. These 12 fibres were not identifiable before the actual splitting.

3. The extent and complexity of the splitting varied considerably from a simple separation and rejoining in three fibres to a multiple, complex separation and recombination seen in three other fibres. The best examples of these are shown in Figs 2, 3, and 4.

4. In the six remaining fibres, daughter fibres and smaller fragments were seen to branch off 


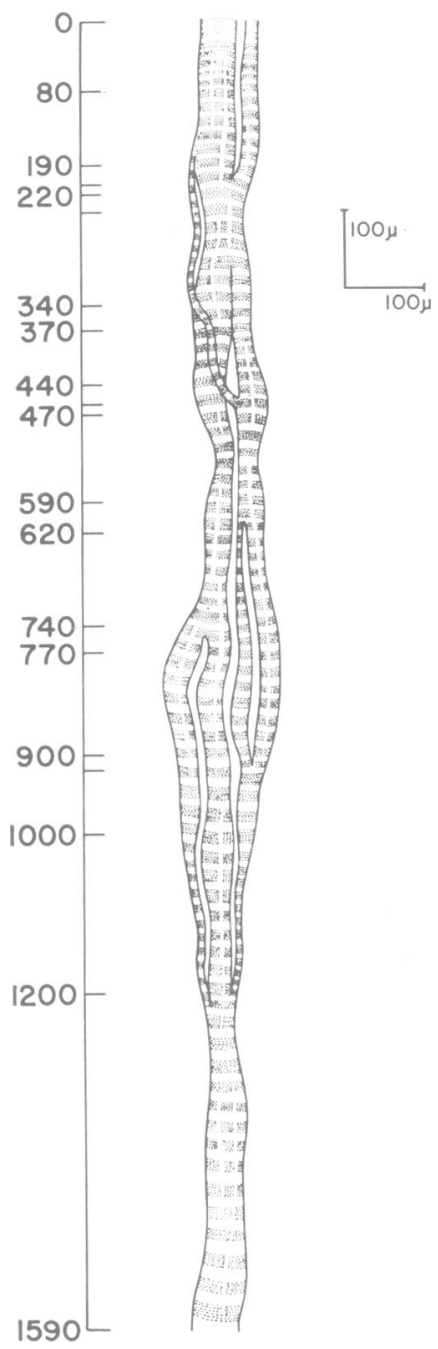

FIG. 3. Fibre reconstructed from serial sections demonstrating a pattern of complex splitting and recombination. The key points in the reconstructed fibre are indicated to the left and correspond to the crosssectional views in Fig. 2.

and extend as separate fibres 50 to $1,400 \mu$ in length. The smaller fragments, $10 \mu$ in diameter would often taper and terminate without rejoining. The larger daughter fibres, 20 to $50 \mu$ in diameter would persist without rejoining.

5. On four occasions, fibres were seen to split several times into four or more segments and
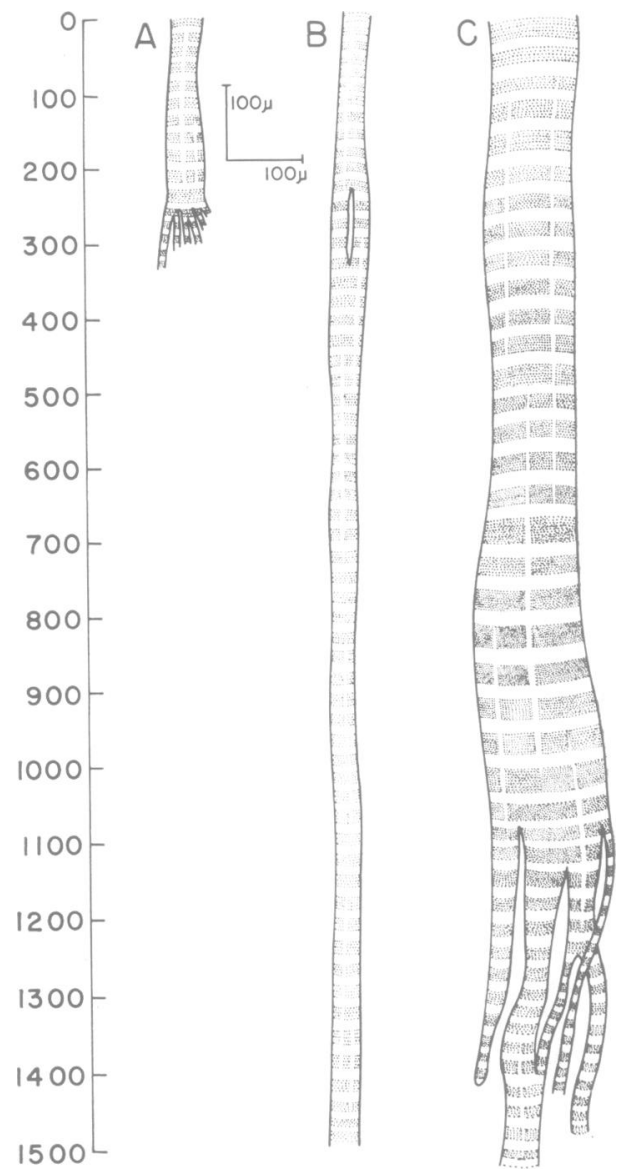

FIG. 4. Reconstructed fibres by the same method used in Fig. 3. A. Fibre splitting into a group of small fibres. Key points indicated to left. B. Simple splitting $\stackrel{\mathrm{Q}}{\mathrm{Q}}$ and recombination. C. More complex splitting and $\overrightarrow{\vec{F}}$ recombination.

appear as a small group of small fibres (Figs $4 \mathrm{~A} \frac{\overline{\bar{\sigma}}}{0}$ and 5).

6. Eight other fibres were seen either to taper off or end abruptly.

\section{DISCUSSION}

The apparatus as assembled for this preliminary? study had some draw-backs. Viewing was confined to a rather small area of muscle. The heat from the 250 watt bulb caused some expansion and softening of the paraffin block despite con-w 

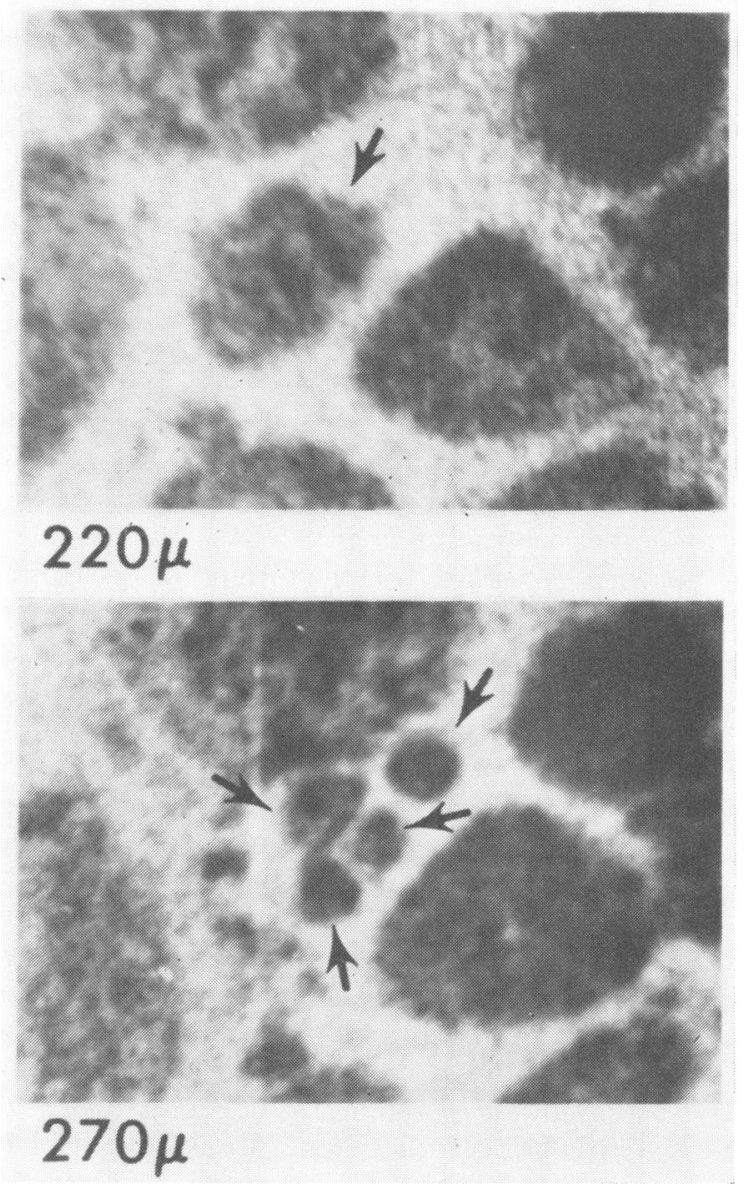

FIG. 5. Actual cross-sectional views corresponding to key points in Fig. 4 (A) showing fibre before and after splitting demonstrating the resemblance to grouped atrophy. tinuous cooling. The most serious handicap was the inability to distinguish endomysial sheath, sarcolemmal nuclei or basophilia with this quick, surface-staining technique. Despite these restrictions, certain advantages became apparent. It was quite easy to remain focused on individual muscle fibres section after section since the movement caused by cutting was minimal. In reviewing the film or enlarged prints, these fibres could be rapidly identified in as many as 200 consecutive sections $(2.0 \mathrm{~mm})$ even if their shapes varied and they migrated to new positions within the fasciculus. The difficulties encountered in analysing hundreds of serial sections by more standard techniques were thereby minimized.

The random variation in muscle fibre diameters is often stressed as a 'myopathic feature' in diseased muscle (Banker, 1960; West and Murphy, 1960; Adams et al., 1962; Pearce and Walton, 1963; Hudgson, Pearce, and Walton, 1967). The presence of hypertrophied fibres is in part responsible for this variation (Banker and Denny-Brown, 1959). Yet, there is a high correlation between the degree of variation in size and the incidence of splitting (Bell and Conen, 1968). While reviewing these serial sections, many daughter fibres were seen to migrate away from the parent fibre. Ordinarily, with a single section, no association between these two fibres would be made. Splitting was therefore seen to be an important contributor to the evolution of the smaller fibres and this work confirms the data obtained by statistical analysis (Bell and Conen, 1968). 
In the past, the question has been raised as to whether or not groups of smaller fibres seen in dystrophic muscle represent the end result of splitting, atrophy of individual fibres or possibly a neurogenic component (Banker, 1960). In this study, several fibres were seen to split one or more times into small groups of daughter fibres of similar diameters, and then migrate short distances away from each other. They then resembled a small group of denervated fibres (Fig. 5). Therefore small groups of atrophic fibres, even of the same fibre-type cannot be accepted as diagnostic of denervation.

The most fascinating aspect of this study was the complex splitting and recombinations seen in several fibres. Mechanical 'overloading' of remaining fibres in dystrophic muscle might be responsible for such splitting. However, what is known about regeneration following a focal necrotizing lesion such as a crush (Clark, 1946; Mauro, Shafiq, and Milhorat, 1970) suggests a more plausible explanation. An area of focal necrosis and phagocytosis promotes regeneration by the release of mononuclear myoblasts which later fuse to form new muscle fibres (DennyBrown, 1960; Adams et al., 1962) and possibly by budding from the ends of intact fibres (Clark, 1946). The mononuclear myoblasts fuse to form myotubes, which often appear multiple though derived from an originally single muscle fibre. Thus several daughter fibres may regenerate from one necrotic fibre.

These multiple daughter fibres may unite at either end of the regenerated segment with the original parent fibre producing the appearance shown in Fig. 3. They may also unite with adjacent regenerating fibres forming cross-bridges between two or more previously individual fibres. In this randomly selected $1.5 \mathrm{~mm}$ sample of muscle, in which 64 fibres were studied, two groups of fibres were seen where there may have been such complex bridging between originally separated parent fibres. This becomes quite significant when an estimate is made of the incidence of such cross-bridging in the entire length of mouse gracilis muscle, which is about $20 \mathrm{~mm}$. By extrapolation over 80 per cent of the fibres might be expected to show cross-bridging at some point along this length. We have not serially sectioned the muscle from one end to the other, and thus cannot do more than state that the effect of such possible cross-bridging formation should be considered when comparing the morphological with the electrophysiological characteristics of dystrophic muscle. The process requires to be studied more extensively and muscle from patients with Duchenne type muscular dystrophy must be examined by a similar technique.

The presence of simple splitting, fibres united $\underset{\gtrless}{\gtrless}$ by cross-bridges and fibres which end abruptly, $\overrightarrow{\vec{F}}$ taper off, or fragment as described here may help to explain some of the electrophysiological findings in muscular dystrophy (McComas and $\frac{\bar{\sigma}}{\bar{n}}$ żek, 1967; McComas, Sica, and Currie, 1970; Harris and Wilson, 1971). They may provide another viewpoint in the debate on the neuropathic hypothesis of the origin of dystrophic muscle. The fibres which ended blindly may be $\overrightarrow{\vec{\omega}}$ separated from the segments containing the motor end plate thereby becoming isolated, functionally denervated and capable of generating fibrillation potentials. This may explain the findings of McComas and Mrożek (1967), and of fibrillation potentials in muscular dystroph which are often taken as evidence of a neurge lesion. Fifteen per cent of the fibres in this pre liminary study were possible sources for sueh spontaneous electrical activity. The isolation $\frac{9}{0}$ ? segments of fibres would reduce the total volume of fibres within a motor unit thereby decreasis the amplitude of the motor unit potential.

Cross-bridges between fibres might have several electrophysiological effects. Conduction of the muscle action potential along the narrow split fibres or cross-bridges might be expected to be slower than normal, contributing to the $\stackrel{\square}{\square}$ appearance of the low voltage polyphasic poten- $\overrightarrow{\overrightarrow{0}}$ tials seen on the electromyogram. If two fibres 3 joined by a cross-bridge retain their original innervation, potentially both might be activated by either nerve fibre. If the nerve to one of the fibres was prevented from acting, either by isola-? tion by a necrotic segment of muscle, or by degeneration as a result of superneurotization, then one nerve fibre would have taken over two muscle fibres. There would in effect be a reorganization of the motor unit caused by the incorporation of muscle fibres by the process of cross-bridge anastomosis.

Although the possibility that neuropathic N factors contribute to the pathophysiology of 0 muscular dystrophy cannot be excluded by this 
study, the morphological changes shown here warrant close scrutiny for further interpretation in this area.

One of us (E.R.I.) wishes to thank Professor Cary Suter, M.D., Medical College of Virginia, Richmond, Virginia, U.S.A. and Professor J. N. Walton, the University of Newcastle upon Tyne, for arranging his exchange. Financial support was provided by grants from the Medical Research Council of Great Britain, the Muscular Dystrophy Group of Great Britain and the Muscular Dystrophy Associations of America, Inc. and by NINDS Graduate Training Grant ST1-NB5490-NSRA.

\section{REFERENCES}

Adams, R. D., Denny-Brown, D., and Pearson, C. M. (1962). Diseases of Muscle: A Study in Pathology. 2nd edn. Harper and Row: New York.

Banker, B. Q. (1960). The experimental myopathies. Research Publications, Association for Research in Nervous and Mental Disease, 38, 197-233.

Banker, B. Q., and Denny-Brown, D. (1959). A study of denervated muscle in normal and dystrophic mice. Journal of Neuropathology and Experimental Neurology, 18, 517530.

Bell, C. D., and Conen, P. E. (1968). Histopathological changes in Duchenne muscular dystrophy. Journal of the Neurological Sciences, 7, 529-544.

Cazzato, G. (1970). Myopathic changes in denervated muscle. A study of biopsy material in various neuromuscular diseases. In Muscle Diseases, pp. 392-401. Proceedings of an International Congress, Milan, 1969. Edited by J. N. Walton, N. Canal, and G. Scarlato. International Congress Series No. 199. Excerpta Medica: Amsterdam.

Clark, W. E. Le G. (1946). An experimental study of the regeneration of mammalian striped muscle. Journal of Anatomy, 80, 24-36.

Denny-Brown, D. (1960). Experimental studies pertaining to hypertrophy, regeneration and degeneration. Research Publications, Association for Research in Nervous and Mental Disease, 38, 147-196.
Drachman, D. B., Murphy, S. R., Nigam, M. P., and Hills, J. R. (1967). 'Myopathic' changes in chronically denervated muscle. Archives of Neurology, 16, 14-24.

Edgerton, V. R. (1970). Morphology and histochemistry of the soleus muscle from normal and exercised rats. American Journal of Anatomy, 127, 81-87.

Hall-Craggs, E. C. B. (1970). The longitudinal division of fibres in overloaded rat skeletal muscle. Journal of Anatomy, 107, 459-470.

Harris, J. B., and Wilson, P. (1971). Mechanical properties of dystrophic mouse muscle. Journal of Neurology, Neurosurgery, and Psychiatry, 34, 512-520.

Hegre, E. S. (1951). A new research tool and technique for the biologist. Virginia Journal of Science, 2, 10-12.

Hegre, E. S. (1967). Serial section cinematography. In Stereology: Proceedings of the Second International Congress for Stereology, Chicago, 1967, pp. 316-322. Springer: Berlin.

Hegre, E. S., and Brashear, A. D. (1947). The block-surface method of staining as applied to the study of embryology. Anatomical Record, 97, 21-28.

Hudgson, P., Pearce, G. W., and Walton, J. N. (1967). Preclinical muscular dystrophy: histopathological changes observed on muscle biopsy. Brain, 90, 565-576.

Mauro, A., Shafiq, S. A., and Milhorat, A. T. (1970). Regeneration of Striated Muscle, and Myogenesis. Proceedings of the International Conference Convened by Muscular Dystrophy Associations of America, New York, 1969. International Congress Series No. 218, Excerpta Medica: Amsterdam.

McComas, A. J., and Mrożek, K. (1967). Denervated muscle fibres in hereditary mouse dystrophy. Journal of Neurology, Neurosurgery, and Psychiatry, 30, 526-530.

McComas, A. J., Sica, R. E. P., and Currie, S. (1970). Muscular dystrophy: evidence for a neural factor. Nature, 226, 1263-1264.

Pearce, G. W., and Walton, J. N. (1963). A histological study of muscle from the Bar Harbor strain of dystrophic mice. Journal of Pathology and Bacteriology, 86, 25-33.

Pearce, J., and Harriman, D. G. F. (1966). Chronic spinal muscular atrophy. Journal of Neurology, Neurosurgery, and Psychiatry, 29, 509-520.

Read, J. L., Hegre, E. S., and Russi, S. (1953). Reaffirmation of the auriculoventricular conduction system in man; the introduction of a unique technic for its serial motion picture reconstruction. Circulation, 7, 42-51.

West, W. T., and Murphy, E. D. (1960). Histopathology of hereditary, progressive muscular dystrophy in inbred strain 129 mice. Anatomical Record, 137, 279-295. 